\title{
Laue 衍射强度分析
}

\author{
张建 中 \\ (南京大学物理系)
}

关链词 Laue 衍射、积分强度、Laue 班点

Laue 法是 X 射线晶体衍射的一个经典方法. 它利用人射光中的连续谱迎合衍射条件产 生衍射.

小晶体的 Laue 衍射积分强度 (或积分功率*)可用运动学衍射理论处理. 早年 Zacharia$\operatorname{sen}^{[1]} 、$ Китайгородский ${ }^{[22]}$ 和近年加藤篹夫 ${ }^{[3]}$ 等人进行过理论计算, 但推导比较抽象, 不易理 解. 而人们对这一重要衍射的强度问题也较陌生, 很少探讨其物理意义和应用前景.

本文根据倒易空间的概念，用简明直观的方法导出 Laue 衍射积分强度公式，讨论了它的 物理意义, 分析了 Laue 斑点的强度组成并探讨应用这种衍射强度的可能性.

\section{一、Laue 衍射积分强度计算}

当单晶试样既不周转也不回摆,而人射 $\mathrm{X}$ 射线又是平行单色光时,能产生衍射的机会是很 少的. 用倒易空间的概念来说, 就是倒易格点不一定正巧落在 Ewald 球面上. 有一个办法可 以使衍射的机会增加一一变化人射线的波长, 采用连续辐射使 Bragg 条件得到满足, 这就是 Laue 衍射的原理. Laue 法中唯一变化的参量是波长,因而积分强度定义为

$$
\mathscr{I} \equiv \int_{2} I d \lambda,
$$

式中 $I$ 为某一波长 $\lambda$ 引起的 $h k l$ 衍射理论强度. 由于晶体有一定大小, 在倒易空间中相应的 $h k l$ 倒易格点弥散为一倒易体积 (又称为选择反射区), 当它和半径为 $\frac{1}{2}$ 的 Ewald 球面相交 时,整个交截面 $\sigma$ 都被激发引起衍射. 设交面 $\sigma$ 时 Ewald 球心 $L$ (Laue 点)所张的立体角为 $\boldsymbol{\rho}$ (图 1), 则

$$
1-\int_{Q} I_{\xi} d Q,
$$

式中 $I_{\xi}$ 为某一衍射束强度, 它与倒易点阵位矢 $\boldsymbol{\xi}-\xi_{1} \boldsymbol{a}^{*}+\xi_{2} \boldsymbol{b}^{*}+\xi_{3} \mathbf{c}^{*}$ 有关, 并定义为单位 立体角中所辐射的功率 ${ }^{[4 b]} . d \Omega$ 为交面上 $d \sigma$ 面元所张的立体角元:

$$
d O-d \sigma /\left(\frac{1}{\lambda}\right)^{2}=\lambda^{2} d \sigma .
$$

在严格满足衍射条件的方向上, 衍射束强度有极大值 ${ }^{(321}$

$$
I_{M}-I_{e} \cdot\left|F_{a t l}\right|^{2} \cdot N^{2} \text {, }
$$

本文1989年 4 月 21 日收到.

- 在晶体行射术语中习惯称行射的能量或功率为强度, 这是不厂格的, 但已沿用成俗 ${ }^{[01}$. 


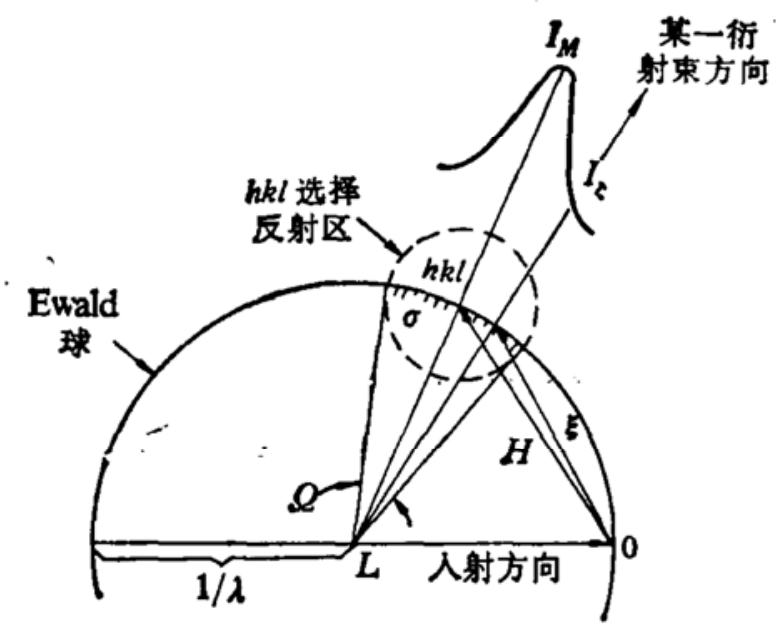

图 1

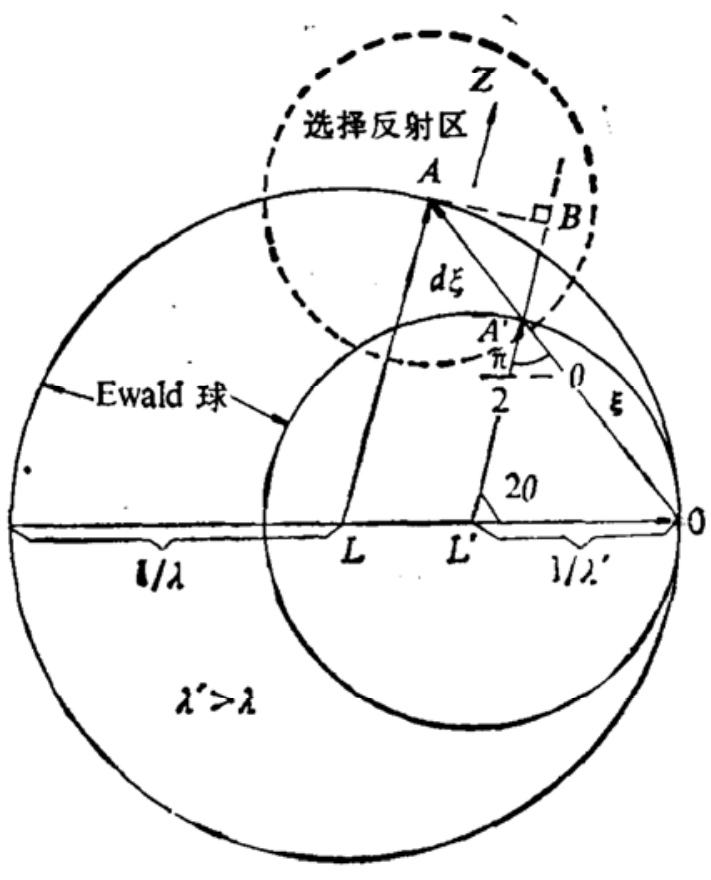

图 2

其中 $I_{e}$ 为电子的 Thomson 散射强度, $F_{k k l}$ 为结构因数, $N$ 为晶体中的晶胞数. 此时倒易点 阵位矢 $\boldsymbol{\xi}$ 等于倒易点阵矢量 $\boldsymbol{H}\left(-h \boldsymbol{a}^{*}+\boldsymbol{k} \boldsymbol{b}^{*}+l \boldsymbol{c}^{*}\right)$. 在极大值附近, 衍射束强度为

$$
I_{\xi}-I_{e} \cdot\left|F_{A k l}\right|^{2} \cdot K \text {, }
$$

式中

$$
K-\prod_{i} \frac{\sin ^{2} \pi N_{i} \xi_{j}}{\sin ^{2} \pi \xi_{i}},(j-1,2,3)
$$

称为 $h k l$ 行射的干涉函数或形状函数 ${ }^{[4 c]} . N_{1} N_{2} N_{3}$ 为沿晶体的 $\alpha b c$ 三个基矢方向的晶胞数 目, $N=N_{1} \cdot N_{2} \cdot N_{3}$. 根据 Bragg 定律和倒易点阵矢量的性质

$$
\frac{2 \sin \theta}{2}-\frac{1}{d}-H \text {, ( } d \text { 为晶面间距、 } \theta \text { 为 Bragg 角) }
$$

在 $h k l$ 点附近, $\xi \simeq H$, 于是

$$
d \xi \simeq-\frac{2 \sin \theta}{\lambda^{2}} d \lambda
$$

式中负号的物理意义是: 波长增加, Ewald 球半径 $\frac{1}{\lambda}$ 减小, 导致 $\boldsymbol{\xi}$ 长度缩短, 如图 2 所示. 因此 (1)式积分强度的物理意义是: 对各种波长引起的衍射强度蔂加; 在倒易空间中相当于波 长变化, Ewald 球面扫过整个选择反射区. 当波长由 $\lambda \rightarrow \lambda+d \lambda$ 时, $A$ 处 Ewald 球面上的 面元 $d \sigma$ 沿球面法线 $\mathrm{Z}$ 方向移动的距离大小为

$$
d Z=B A^{\prime}-|d \xi| \cdot \sin \theta,
$$

把 (7) 式代人到上式中可得到

$$
d \lambda-\frac{\lambda^{2}}{2 \sin ^{2} \theta} d Z
$$

利用(2)、(3)、(8)式的关系，(1)式可化为 


$$
\mathscr{I}=\frac{\lambda^{4}}{2 \sin ^{2} \theta} \int_{0} I_{\xi} d v^{*},
$$

式中 $d v^{*}-d \sigma d Z$, 为 Ewald 球面扫过的倒易体积元, $v^{*}$ 为选择反射区体积. (9) 式表明衍 射的积分强度和带有强度权重的倒易体积积分 $\int I_{\xi} d v^{*}$ 有关. 这个积分纯由倒易体积本身引 起, 与衍射方法无关, 称为理论积分强度. 它适用于各种衍射方法 (Laue 法、粉末法、转晶法) 的积分强度计算, 有普遍意义. 这里不妨称为特征积分 $I_{c}$

$$
I_{c}=\int_{, *} I_{\xi} d v^{*},
$$

把(5)代人上式并注意 $d v^{*}=\left(\boldsymbol{a}^{*} \cdot \boldsymbol{b}^{*} \times \boldsymbol{c}^{*}\right) \cdot d \xi_{1} \cdot d \xi_{2} \cdot d \xi_{3}=\frac{1}{V} \cdot d \xi_{1} \cdot d \xi_{2} \cdot d \xi_{3}(V$ 为晶 胞体积), 所以

$$
I_{c}=I_{e} \cdot \frac{\mid F_{\left.k k_{1}\right|^{2}}}{V} \cdot \prod_{i} \int \frac{\sin ^{2} \pi N_{i} \xi_{i}}{\sin ^{2} \pi \xi_{i}} d \xi_{i},
$$

上式中积分在 $h k l$ 倒易格点周围进行. 根据点阵的性质: 所有的倒格点相同, 它周围的环境 也相同, 因此可在倒易点阵原点附近完成这一积分. 在 $\xi=0$ 的附近, $\boldsymbol{\xi}$ 很小, (11)式中的积分 可简化为

$$
\int \frac{\sin ^{2} \pi N_{i} \xi_{j}}{\left(\pi \xi_{i}\right)^{2}} d \xi_{i}
$$

当 $\xi_{i} \uparrow$ 时, 被积函数迅速衰减,因此积分限可扩展为 $-\infty \rightarrow+\infty$, 于是

$$
\int_{-\infty}^{\infty} \frac{\sin ^{2} \pi N_{i} \xi_{i}}{\left(\pi \xi_{i}\right)^{2}} d \xi_{i}-N_{i}
$$

所以特征积分 (10)式为

$$
I_{c}-I_{e} \cdot \frac{\left|F_{A k l}\right|^{2}}{V} \cdot N
$$

根据 Guinier ${ }^{[5 b]}$, 平均一个晶胞对理论积分强度的贡献正是 $I_{e} \cdot \frac{\left|F_{\Delta k l}\right|^{2}}{V}$. 用晶体体积 $\Delta \boldsymbol{V}$ 这个可直接测量的量来表示晶胞数目, $N-\frac{\Delta V}{V}$, 并把电子的 Thomson 散射强度表达 式[s]代人到公式(9)中, 最后可得到 Laue 衍射积分强度公式:

$$
\mathscr{I}=I_{0} \cdot r_{e}^{2} \cdot \lambda^{4} \cdot\left|F_{k k l}\right|^{2} \cdot\left(\frac{1+\cos ^{2} 2 \theta}{4 \sin ^{2} \theta}\right) \cdot \frac{\Delta V}{V^{2}},
$$

式中 $r_{e}-\frac{e^{2}}{4 \pi \varepsilon_{0} m c^{2}}$ 为电子的 Lorentz 半径, 符号 $e 、 \varepsilon_{0} 、 m 、 c$ 与电磁学中通常意义相同. $\frac{1+\cos ^{2} 2 \theta}{\sin ^{2} \theta}-L_{p}$, 称为 Laue 法的 Lorentz-偏振因数.

\section{二、讨 论}

如果进一步考虑温度效应、吸收修正,(13)式就是小晶体 Laue 衍射强度的资用公式, 应 能用于理论计算和实验结果的比较, 实际上有一定困难, 主要是引起 $h k l$ 衍射的波长 $\lambda$ 未知, 而且某一 Laue 点可能含有若千级 $h k l$ 衍射. 事实上, 当衍射级 $n-1$, 波长取 $2, n-2$; 
波长取 $\frac{\lambda}{2}, n-3$; 波长取 $\frac{\lambda}{3} \cdots \cdots$ 时, Bragg 条件

$$
2 d_{n k l} \sin \theta=n \lambda
$$

都能满足,而且各级衍射线有相同的衍射角,导致衍射点重合. 但由 (13) 式知,积分强度和 波长的四次方成正比,故基波 $\lambda$ 引起的一级衍射是主要的. $\frac{\lambda}{2}$ 的二级衍射的贡献约为 $6 \%, \frac{\lambda}{3}$ 的三级衍射约为 $1 \%$, 更高级的衍射可以忽略. 因此, 对 Laue 玨点进行指标化 ${ }^{[2 b]}$ 后, 可根据 衍射斑点的角位置和点阵参数数据来确定基波 $\lambda$ 的大小.

近年来, 新型探测系统如固态探测器 (SSD) 和平面记数装置 (如多丝正比记数器 ${ }^{(6)}$ ) 等 正在发展,传统的 Laue 照相有可能被新一代的辐射探测方法更新. 它们可迅速显示衍射花 样、记录衍射强度并鉴别强度的组成(能谱). 这将为利用 Laue 行射收集强度数据提供诱人 前累。

利用 Laue 方法的多色光研究消光效应和波长的关系一直是行射领域关注的问题. 目前 在中子衍射中已运用所谓“飞行时间”技术 (TOF) 来研究这一现象 ${ }^{[7]}$. 可以期望在 X 射线衍 射领域利用 Laue 衍射强度研究这一问题, 它将比中子衍射更为简便.

\section{考文献}

[1 1] Zachariasen, W. H., Theory of X-Ray Diffraction in Crystals, J. Wiley and Sons, New York, $1945,105$.

[2] Китайгородский А. И., Рентгеноструктурный Амализ, Гостехиздат, 1950; X射线结构分析(中译本), 科 学出版社, 1958 , 2. 224 ; b. 285 .

(3] 加藤篟夫, 回析と散乱, 朝金者店,1978,166.

[4] Woolfson, M. M. An Introduction to X-ray Crystallugraphy, Cambridge University Press, 1978, a, 156; b. 36; c. 55.

[5] Guinier, A., X-ray Diffraction, W. Freeman and :ompany, San Francisco and London, 1963, a, 126; b. 92; a. 11.

[ 6] Cork, C. et al, Acse Cryst, A31(1975), 702.

[7] Nimura, N., J. A. Cryst, 8(1975), 560. 